\title{
Statistical Mechanical Properties of Polymer Configurations which Enclose a Constant Area
}

\author{
D. C. Khandekar ${ }^{1,2}$ and F. W. Wiegel ${ }^{3}$
}

Received May 25, 1988; revision received August 3, 1988

\begin{abstract}
The statistical mechanical properties of plane polymer loops enclosing a constant area are investigated, using a continuous model from the start. For this purpose an analytic expression for the generating functional is obtained, which in turn is used to derive (1) the distribution function for the enclosed area, (2) the average squared distance of a given repeating unit from the origin, and (3) the entropic force on a repeating unit.
\end{abstract}

KEY WORDS: Polymers; path integrals; constraints.

\section{INTRODUCTION}

Many physical properties of concentrated polymeric systems are due to the mutual entanglements of these chainlike molecules. Although the theory of polymeric systems is now coming to maturity (see the recent monograph by Doi and Edwards ${ }^{(1)}$ ), the analytical treatment of topological entanglements is still unsatisfactory. The mathematical description of various simple entanglements, as well as of knots and links, was recently reviewed by Wiegel. ${ }^{(2)} \mathrm{A}$ new way to work directly in covering space forms the subject of ref. 3 and the exactly solvable cases involving constraints were discussed in ref. 4 . In all these exactly solvable models the number of polymers was restricted to 1 or 2 , and no exact solutions exist for the case in which many polymers are involved.

\footnotetext{
${ }^{1}$ Research Center BiBoS, University of Bielefeld, 4800 Bielefeld 1, Federal Republic of Germany.

${ }^{2}$ Permanent address: Theoretical Physics Division, Bhabha Atomic Research Centre, Bombay 400085 , India.

${ }^{3}$ Center for Theoretical Physics, Twente University, Enschede 7500 AE, The Netherlands.
} 
It is therefore of interest to simulate the topology of entangled polymers in a variety of ways. Recently, Brereton and Butler ${ }^{(5)}$ suggested a new way to do this: (1) represent a polymer by a random walk of $N$ steps in a plane; each step has length $l$; (2) approximate the steric and topological effects of the other polymers in the system by the constraint that this walk encloses a fixed (algebraic) area. In view of the simplicity of this model for entangled polymers, crude as it might be, we have used it to calculate a variety of statistical properties of the configurations. In doing this we have used a continuous model from the start, as reported in ref. 6 .

\section{THE GENERATING FUNCTION}

It will turn out that all quantities of interest can be derived from a generating function $Z$ which is defined by the path integral

$$
\begin{aligned}
Z(A)= & \int_{\mathbf{r}^{\prime}, 0}^{\mathrm{r}^{\prime \prime}, N} \delta\left(A-\frac{1}{2} \int_{0}^{N}\left(x \frac{d y}{d v}-y \frac{d x}{d v}\right) d v\right) \\
& \times \exp \left[\boldsymbol{\eta} \cdot \mathbf{r}\left(N^{\prime}\right)-\frac{1}{l^{2}} \int_{0}^{N}\left(\frac{d \mathbf{r}}{d v}\right)^{2} d v\right] d[\mathbf{r}(\nu)]
\end{aligned}
$$

In this formula the notation of ref. 2 is used for the path integral over all paths (polymer configurations) in the plane which start at $\mathbf{r}^{\prime}$ and end in $\mathbf{r}^{\prime \prime}$. The vector $\boldsymbol{\eta}$ is an auxiliary variable, and $0<N^{\prime}<N$. The delta function in the integrand limits the paths to those for which the enclosed algebraic area equals

$$
A=\frac{1}{2} \int_{0}^{N}\left(x \frac{d y}{d v}-y \frac{d x}{d v}\right) d v
$$

The use of this formula requires some words of caution.

1. The phrase "enclosed area" in practice ceases to be physically meaningful for open chains. However, one can define the algebraic area of an open chain as the algebraic area of the loop closed by joining the two endpoints by a straight line. The algebraic area $A$ for a closed curve, on the other hand, is meaningful both physically and mathematically.

2. The expression (2.2) is the symmetrical form of the two expressions

$$
A=\int x d y \quad \text { and } \quad A=-\int y d x
$$

3. If the closed loop has no double points, then (2.2) equals the geometrical area, where the plus sign obtains if the enclosed area is always 
situated to the left of the polymer configuration and the minus sign obtains if the opposite holds. If the closed loop has one or more double points, the algebraic area $(2.2)$ is not exactly equal to the geometrical area, because some "dangling" loops will be counted with the wrong sign; moreover, some subareas will be counted by $(2.2)$ more than once.

4. For self-avoiding plane polymer configurations (which we shall not study in this paper) the loops cannot have any double points, hence eq. (2.2) is identical to the geometrical area, apart from the sign. Such configurations were recently studied by Leibler et al. ${ }^{(7)}$ in a slightly different context.

The three main quantities of physical interest and their relation to the generating function are as follows:

(a) The probability density $P(A, N)$ that a closed polymer configuration will enclose an algebraic area $A$ is given by

$$
P(A, N)=\left(\pi N l^{2}\right) Z \quad \text { with } \quad\left(\mathbf{r}^{\prime}=\mathbf{0}, \mathbf{r}^{\prime \prime}=\mathbf{0}, \boldsymbol{\eta}=\mathbf{0}\right)
$$

(b) The average $\left\langle\mathbf{r}^{2}\left(N^{\prime}\right)\right\rangle$ of the position of repeating unit $N^{\prime}$, squared, when both endpoints are fixed at the origin, is given by

$$
\left\langle\mathbf{r}^{2}\left(N^{\prime}\right)\right\rangle=\left(\frac{1}{Z} \frac{\partial}{\partial \boldsymbol{\eta}} \cdot \frac{\partial}{\partial \boldsymbol{\eta}} Z\right) \quad \text { with } \quad\left(\mathbf{r}^{\prime}=\mathbf{0}, \mathbf{r}^{\prime \prime}=\mathbf{0}, \boldsymbol{\eta}=\mathbf{0}\right)
$$

(c) The configuration sum density $\Omega(A, \mathbf{R})$ over all those polymer configurations which span an area equal to $A$ and for which $\mathbf{r}\left(N^{\prime}\right)-\mathbf{r}(0)=\mathbf{R}$ is given by

$$
\Omega(A, \mathbf{R})=\frac{1}{2} N l^{2} \int Z(\boldsymbol{\eta}=-i \zeta) \exp (i \zeta \cdot \mathbf{R}) d^{2} \zeta
$$

In order to proceed with the evaluation of the generating function, one represents the delta function in the integrand of $(2.1)$ by a Fourier integral, which gives

$$
\begin{aligned}
& Z(A)=\frac{1}{2 \pi} \int_{-\infty}^{\infty} \tilde{Z}(g) \exp (i g A) d g \\
& \tilde{Z}(g)=\int_{\mathbf{r}^{\prime}, 0}^{r^{\prime \prime}, N} \exp \left[-\int_{0}^{N} L d v+\boldsymbol{\eta} \cdot \mathbf{r}\left(N^{\prime}\right)\right] d[\mathbf{r}(v)]
\end{aligned}
$$

The "Langrangian" is given by

$$
L=\frac{1}{l^{2}}\left(\frac{d \mathbf{r}}{d v}\right)^{2}+\frac{i}{2} g\left(x \frac{d y}{d v}-y \frac{d x}{d v}\right)
$$


As the second term $\boldsymbol{\eta} \cdot \mathbf{r}\left(N^{\prime}\right)$ in the exponent of (2.7) depends on the position of repeating unit $N^{\prime}$ only, it is straightforward to perform the path integral over all other positions and to show that

$$
\tilde{Z}(g)=\int G\left(\mathbf{r}^{\prime \prime}, N \mid \mathbf{r}, N^{\prime}\right) \exp (\boldsymbol{\eta} \cdot \mathbf{r}) G\left(\mathbf{r}, N^{\prime} \mid \mathbf{r}^{\prime}, 0\right) d^{3} \mathbf{r}
$$

The function $G$ was calculated in ref. 6 and found to be given by

$$
\begin{aligned}
& G\left(x_{1}, y_{1}, N_{1} \mid x_{0}, y_{0}, 0\right) \\
&=\frac{\omega_{0}}{2 \pi l^{2} \sin \left(\frac{1}{2} N \omega_{0}\right)} \\
& \times \exp \left\{\frac{\omega_{0}}{l^{2}}\left(x_{0} y_{1}-x_{1} y_{0}\right)-\frac{\omega_{0}}{2 l^{2}}\left[\left(x_{1}-x_{0}\right)^{2}+\left(y_{1}-y_{0}\right)^{2}\right]\right. \\
&\left.\times \operatorname{cotg}\left(\frac{1}{2} N \omega_{0}\right)\right\}
\end{aligned}
$$

where

$$
\omega_{0}=-\frac{1}{2} i g l^{2}
$$

For closed polymers we set $\mathbf{r}^{\prime}=\mathbf{r}^{\prime \prime}=\mathbf{0}$ in Eqs. (2.9) and (2.10); this is convenient and physically interesting, but does limit the generality of the results (similar results can be derived for open chains). The resulting integral

$$
\begin{aligned}
\tilde{Z}_{c}(g)= & \left(\frac{\omega_{0}}{2 \pi l^{2}}\right)^{2}\left\{\sin \left(\frac{1}{2} N^{\prime} \omega_{0}\right) \sin \left[\frac{1}{2}\left(N-N^{\prime}\right) \omega_{0}\right]\right\}^{-1} \\
& \times \int \exp \left\{\boldsymbol{\eta} \cdot \mathbf{r}-\frac{\omega_{0} r^{2}}{2 l^{2}}\left[\operatorname{cotg}\left(\frac{1}{2} N^{\prime} \omega_{0}\right)\right.\right. \\
& \left.\left.+\operatorname{cotg}\left(\frac{1}{2}\left(N-N^{\prime}\right) \omega_{0}\right)\right]\right\} d^{2} \mathbf{r}
\end{aligned}
$$

can be evaluated in a straightforward manner, with the result

$$
\tilde{Z}_{c}(g)=\frac{1}{\pi N l^{2}} \frac{u}{\sinh u} \exp \left[\frac{\phi}{u} \frac{\sinh \alpha u \sinh (1-\alpha) u}{\sinh u}\right]
$$

where we introduced the dimensionless scaled quantities

$$
\begin{aligned}
& u \equiv \frac{1}{4} N g l^{2} \\
& \phi \equiv \frac{1}{4} N \eta^{2} l^{2} \\
& \alpha \equiv N^{\prime} / N
\end{aligned}
$$


The generating function $Z_{c}(A)$ for a closed polymer is found upon substitution of (2.13) into (2.6)

$$
Z_{c}(A)=\frac{2}{\left(\pi N l^{2}\right)^{2}} \int_{-\infty}^{\infty} \frac{u}{\sinh u} \exp \left[i u \xi+\frac{\phi}{u} \frac{\sinh \alpha u \sinh (1-\alpha) u}{\sinh u}\right] d u
$$

where

$$
\xi \equiv 4 A / N l^{2}
$$

This completes the analytic calculation of the generating functional.

\section{CONFIGURATIONAL PROPERTIES}

In this section we calculate the three quantities of interest quoted under $\mathrm{a}, \mathrm{b}$ and $\mathrm{c}$ in Section 2 . The probability density $P(A, N)$ that a closed polymer configuration will enclose an algebraic area $A$ is found from (2.3) and $(2.15)$ to be given by

$$
P(A, N)=\frac{2}{\pi N l^{2}} \int_{-\infty}^{\infty} \frac{u}{\sinh u} \exp (i u \xi) d u=\left\{2 N l^{2} \cosh ^{2}\left(\frac{2 \pi A}{N l^{2}}\right)\right\}^{-1}
$$

which agrees with the result found in ref. 6.

The average $\left\langle\mathbf{r}^{2}\left(N^{\prime}\right)\right\rangle$ of the position (squared) of repeating unit $N^{\prime}$, with both end points fixed at the origin, is found by combination of (2.4), (2.15) and (3.1)

$$
\left\langle\mathbf{r}^{2}\left(N^{\prime}\right)\right\rangle=\frac{4 N l^{2}}{\pi} \cosh ^{2}\left(\frac{2 \pi A}{N l^{2}}\right) \int_{-\infty}^{\infty} \frac{\sinh \alpha u \sinh (1-\alpha) u}{\sinh ^{2} u} \exp (i u \xi) d u
$$

The integral is evaluated in the appendix, with the result

$$
\begin{aligned}
\left\langle\mathbf{r}^{2}\left(N^{\prime}\right)\right\rangle= & 4 N l^{2} \cosh ^{2}\left(\frac{2 \pi A}{N l^{2}}\right) \\
& \cdot\left[\frac{\xi e^{-x}}{1+e^{-x}}=\frac{\xi e^{-x}\left(e^{-x}+\cos y\right)-(1-2 \alpha) e^{-x} \sin y}{1+2 e^{-x} \cos y+e^{-2 x}}\right]
\end{aligned}
$$

where

$$
\begin{aligned}
& x=\pi \xi=\frac{4 \pi A}{N l^{2}} \\
& y=2 \pi \alpha=2 \pi \frac{N^{\prime}}{N}
\end{aligned}
$$


It is immediately clear from (3.3) that $\left\langle\mathbf{r}^{2}\left(N^{\prime}\right)\right\rangle$ is symmetric under the change $\alpha \leftrightarrow 1-\alpha$. It is also of some interest to examine the limiting behaviour for small and large values of $\alpha$ and $\xi$ respectively. First, note that for small $\alpha$

$$
\left\langle\mathbf{r}^{2}\left(N^{\prime}\right)\right\rangle \cong 2 \pi l^{2} N^{\prime}\left(1-2 \frac{N^{\prime}}{N}\right), \quad(\alpha \ll 1 / 2)
$$

which is the result for an unconstrained random walk. In the opposite case, where $\alpha=1 / 2$, one finds

$$
\left\langle\mathbf{r}^{2}(N / 2)\right\rangle=8 A(1+\cosh x) / \sinh x
$$

In this case the constraint imposes a kind of rigidity on the overall shape of the polymer, which might have been expected to begin with.

The third quantity of interest quoted in II is the configuration sum density $\Omega(A, \mathbf{R})$. Substituting (2.15) into (2.5), one finds

$$
\begin{aligned}
\Omega(A, \mathbf{R})= & \frac{1}{\pi^{2} N l^{2}} \int \exp (i \zeta \cdot \mathbf{R}) d^{2} \zeta \\
& \times \int_{-\infty}^{\infty} \frac{u}{\sinh u} \exp \left[i u \xi-\frac{N l^{2}}{4} \zeta^{2} \frac{\sinh \alpha u \sinh (1-\alpha) u}{u \sinh u}\right] d u
\end{aligned}
$$

Carrying out the integration over $\zeta$, one finds

$$
\begin{aligned}
\Omega(A, \mathbf{R})= & \frac{4}{\pi\left(N l^{2}\right)^{2}} \int_{-\infty}^{+\infty} \frac{u^{2}}{\sinh \alpha u \sinh (1-\alpha) u} \\
& \times \exp \left[i u \xi-\frac{R^{2}}{N l^{2}} \frac{u \sinh u}{\sinh \alpha u \sinh (1-\alpha) u}\right] d u
\end{aligned}
$$

The Helmholtz free energy is given by

$$
F(A, R)=-k T \ln \Omega
$$

where $k$ denotes Boltzmann's constant and $T$ the absolute temperature. The physical quantity which most clearly shows the effects of the constraint is the entropic force on repeating unit $N^{\prime}$. It is given by

$$
\mathbf{f}(A, R)=-\nabla F=\frac{k T}{\Omega} \nabla \Omega
$$


or, more explicitly,

$$
\begin{aligned}
\mathbf{f}(A, R)= & -\frac{8 k T}{\pi\left(N l^{2}\right)^{3} \Omega} \mathbf{R} \int_{-\infty}^{\infty} \frac{u^{3} \sinh u}{\sinh ^{2} \alpha u \sinh ^{2}(1-\alpha) u} \\
& \times \exp \left[i u \xi-\frac{R^{2}}{N l^{2}} \frac{u \sinh u}{\sinh \alpha u \sinh (1-\alpha) u}\right] d u
\end{aligned}
$$

Note that this elastic force is nonlinear, which again reflects the presence of the constraint.

\section{APPENDIX. EVALUATION OF AN INTEGRAL}

In this Appendix we evaluate the integral

$$
I \equiv \int_{-\infty}^{\infty} \frac{\sinh \alpha u \sinh (1-\alpha) u}{\sinh ^{2} u} \exp (i u \xi) d u
$$

As $\xi$ is positive, the contour of integration can be closed by half a circle in the upper half of the complex $u$ plane. In the limit in which the radius of this circle tends to $\infty$, one finds

$$
I=2 \pi i \sum_{n=1}^{\infty} R_{n}
$$

where $R_{n}$ equals the residue

$$
\begin{aligned}
R_{n} & =\frac{d}{d u}[\sinh \alpha u \sinh (1-\alpha) u \exp (i u \xi)] \\
& =\frac{1}{2} \frac{d}{d u}\{\exp (i u \xi)[\cosh u-\cosh (1-2 \alpha) u]\}
\end{aligned}
$$

evaluated at $u=u_{n} \equiv n \pi i$. One finds explicitly

$$
R_{n}=\frac{i}{2}(-1)^{n} e^{-n \pi \xi}[\xi(1-\cos 2 \pi n \alpha)+(1-2 \alpha) \sin 2 \pi n \alpha]
$$

and consequently the integral (A.1) is given by

$$
I=-\pi \sum_{n=1}^{\infty} e^{-n \pi \xi}(-1)^{n}[\xi(1-\cos 2 \pi n \alpha)+(1-2 \alpha) \sin 2 \pi n \alpha]
$$

To evaluate the various sums occurring in Eq. (A.5), consider the geometric series

$$
S=\sum_{n=1}^{\infty} \exp [-n(x-i y)](-1)^{n}
$$


The series in (A.6) can easily be evaluated in closed form, leading to

$$
S=-\frac{e^{-(x-i y)}}{1+e^{-(x-i y)}} \equiv-\frac{e^{-2 x}+e^{-x} e^{i y}}{1+2 e^{-x} \cos y+e^{-2 x}}
$$

A comparison of real and imaginary parts of (A.6) with (A.7) immediately yields

$$
\begin{aligned}
& \sum_{n=1}^{\infty}(-1)^{n} e^{-n x} \cos n y=-\frac{e^{-2 x}+e^{-x} \cos y}{1+2 e^{-x} \cos y+e^{-2 x}} \\
& \sum_{n=1}^{\infty}(-1)^{n} e^{-n x} \sin n y=-\frac{e^{-x} \sin y}{1+2 e^{-x} \cos y+e^{-2 x}}
\end{aligned}
$$

Further, when we substitute $y=0$ in (A.8) we obtain

$$
\sum_{n=1}^{\infty}(-1)^{n} e^{-n x}=-\frac{e^{-x}}{1+e^{-x}}
$$

Using (A.8)-(A.10) with the values $x$ and $y$ given by (3.4a), (3.4b), we find for Eq. (A.5)

$$
\begin{aligned}
I= & \pi\left\{\xi\left[\frac{e^{-x}}{1+e^{-x}}-\frac{e^{-x}\left(e^{-x}+\cos y\right)}{1+2 e^{-x} \cos y+e^{-2 x}}\right]\right. \\
& \left.+(1-2 \alpha) \frac{e^{-x} \sin y}{1+2 e^{-x} \cos y+e^{-2 x}}\right\}
\end{aligned}
$$

\section{ACKNOWLEDGMENTS}

One of the authors (D.C.K.) acknowledges the hospitality of the Department of Applied Physics at Twente University during the spring of 1988. D.C.K. is an Alexander von Humboldt fellow.

\section{REFERENCES}

1. M. Doi and S. F. Edwards, The Theory of Polymer Dynamics (Oxford University Press, Oxford, 1986).

2. F. W. Wiegel, Introduciion to Path-Integral Methods in Physics and Polymer Science (World Scientific, Singapore, 1986), Chapter IV.

3. D. C. Khandekar, K. V. Bhagwat, and F. W. Wiegel, Phys. Lett. A 127:379 (1988).

4. D. C. Khandekar and S. V. Lawande, Phys. Rep. 137:115 (1986).

5. M. G. Brereton and C. Butler, J. Phys. A 20:3955 (1987).

6. D. C. Khandekar and F. W. Wiegel, J. Phys. A 21:L563 (1988).

7. S. Leibler, R. R. P. Singh, and M. E. Fisher, Phys. Rev. Lett. 59:1989 (1987). 